\title{
The Neural Sociometer: Brain Mechanisms Underlying State Self-esteem
}

\author{
Naomi I. Eisenberger ${ }^{1}$, Tristen K. Inagaki ${ }^{1}$, Keely A. Muscatell ${ }^{1}$, \\ Kate E. Byrne Haltom ${ }^{1}$, and Mark R. Leary ${ }^{2}$
}

\begin{abstract}
On the basis of the importance of social connection for survival, humans may have evolved a "sociometer"-a mechanism that translates perceptions of rejection or acceptance into state self-esteem. Here, we explored the neural underpinnings of the sociometer by examining whether neural regions responsive to rejection or acceptance were associated with state self-esteem. Participants underwent fMRI while viewing feedback words ("interesting," "boring") ostensibly chosen by another individual (confederate) to describe the participant's previously recorded interview. Participants rated their state self-esteem in response
\end{abstract}

\section{INTRODUCTION}

Walk down the self-help aisle at any bookstore and you will immediately notice dozens of books on the importance of self-esteem and how to improve it-with titles such as Ten Simple Solutions for Building Self-esteem, Believing in Myself: Self-esteem Daily Meditations, and Breaking the Chain of Low Self-esteem. Books like these attest to the intuitive notion that we all want to feel good about ourselves; we all want to have high self-esteem. And this is not a new idea; James (1890), in the first psychology textbook, asserted that striving to feel good about oneself is fundamental to human nature. In the 130 years since his writings, a great deal of psychological research has focused on the motivation to protect and enhance self-esteem as well as the positive correlates of having high self-esteem such as better performance outcomes and greater psychological adjustment (Leary, 1999). But why do we strive not just to feel good but to feel good about ourselves? Why do we care so profoundly about self-esteem?

One account for why people strive for high self-esteem is sociometer theory, which suggests that feelings about the self at any moment in time (state self-esteem) are a direct readout of how individuals believe they are perceived or evaluated by others (Leary, 2006a, 2006b; Leary, Haupt, Strausser, \& Chokel, 1998; Leary \& Downs, 1995).

${ }^{1}$ University of California, Los Angeles, ${ }^{2}$ Duke University, Durham, USA to each feedback word. Results demonstrated that greater activity in rejection-related neural regions (dorsal ACC, anterior insula) and mentalizing regions was associated with lowerstate self-esteem. Additionally, participants whose self-esteem decreased from prescan to postscan versus those whose selfesteem did not showed greater medial prefrontal cortical activity, previously associated with self-referential processing, in response to negative feedback. Together, the results inform our understanding of the origin and nature of our feelings about ourselves.

Given the importance of social relationships for survival, human beings may have developed an internal gauge-a "sociometer"- that monitors the degree to which they are accepted or rejected by others and that results in highor low-state self-esteem (Leary et al., 1998). Thus, although moment-to-moment assessments of self-esteem may feel like a private evaluation of one's self-worth-separate from the opinions and feedback of others-sociometer theory suggests that self-esteem is directly responsive to perceived acceptance or rejection from others. Accordingly, "self-esteem" may actually be more appropriately termed "social esteem."

Consistent with this notion, behavioral research has demonstrated that experimental manipulations of acceptance and rejection reliably influence state self-esteem (Leary, 2006a) and that state self-esteem correlates very highly with perceived acceptance and rejection (Leary, 2006b; Leary, Tambor, Terdal, \& Downs, 1995). Still, the neural underpinnings of these moment-to-moment fluctuations in state self-esteem have not been explored and may provide additional evidence to support the sociometer theory that does not rely solely on self-report. Although several studies have investigated the neural correlates of how trait self-esteem relates to neural responses to evaluative feedback (Somerville, Kelley, \& Heatherton, 2010) and inclusionary status (Onoda et al., 2010), no studies have investigated the neural underpinnings of the sociometerthe mechanism that is hypothesized to translate momentto-moment perceptions of rejection and acceptance into moment-to-moment changes in state self-esteem. To 
the extent that moment-to-moment fluctuations in state self-esteem result from perceptions of rejection or acceptance (Leary et al., 1998), one might expect that neural regions involved in feeling rejected or accepted would also be involved in feelings of state self-esteem.

Along these lines, research has shown that being socially excluded or rejected, compared with being included or accepted, leads to increased activity in the dorsal ACC (dACC) and anterior insula, neural regions that are often associated with the distress of physical pain (DeWall et al., 2010; Eisenberger, Lieberman, \& Williams, 2003). Moreover, higher levels of trait rejection sensitivity are associated with greater activity in these regions in response to rejecting stimuli (Burklund, Eisenberger, \& Lieberman, 2007; Kross, Egner, Ochsner, Hirsch, \& Downey, 2007). In addition, greater dACC and anterior insula activity in response to rejection has been shown to correlate with greater self-reported social distress (Masten et al., 2009; Onoda et al., 2009; Eisenberger, Taylor, Gable, Hilmert, \& Lieberman, 2007; Eisenberger et al., 2003). On the basis of these findings, we hypothesized that greater activity in the dACC and anterior insula in response to evaluative appraisals will relate to lower-state self-esteem because of their role in processing social rejection.

In addition to research on social rejection, another body of work has identified some of the neural regions that are responsive to social acceptance and positive social feedback. Receiving feedback that one is liked by another person activates the subgenual ACC (subACC; Somerville, Heatherton, \& Kelley, 2006), and receiving positive descriptive feedback about oneself activates the ventral striatum (VS; Izuma, Saito, \& Sadato, 2008), a neural region involved in reward processing (Knutson \& Cooper, 2005). Thus, greater subACC or VS activity in response to evaluative appraisals may relate to higher-state self-esteem.

Finally, given that state self-esteem is hypothesized to be responsive to the appraisals of others, neural regions involved in mentalizing-understanding the minds of others-may be critical for understanding the appraisals of others, which may lead to changes in state self-esteem. As such, regions such as the dorsomedial pFC (DMPFC), precuneus, posterior STS (pSTS), and TPJ (Lieberman, 2010; Mitchell, 2009; Frith \& Frith, 2006) may also be recruited in service of translating the perception of others' positive or negative interpersonal appraisals into state self-esteem.

To examine the neural underpinnings of the sociometer, we adapted a behavioral paradigm that has been shown to modulate state self-esteem (Leary et al., 1998). Participants were ostensibly recorded as they completed an interview and were told that another subject would later listen to the audio recording and provide continuous feedback on how the participant was "coming across." Participants then completed an fMRI scan, as they viewed descriptive feedback words (e.g., "interesting," "boring") presented at 10-sec intervals, supposedly chosen by the other subject who was listening to the recorded interview. Participants rated how they felt about themselves in response to each feedback word (state self-esteem). On the basis of the notion that state self-esteem is responsive to the subjective interpretation of the appraisals of others, we examined how state self-esteem correlated with neural activity in response to each feedback word. Finally, to examine possible longer-lasting effects of processing this evaluative feedback, we examined how neural activity during the task itself related to changes in participants' state self-esteem from prescan to postscan (changes in session-level self-esteem).

\section{METHODS}

\section{Participants}

Nineteen participants ( 12 women; $M=20.32$ years, range $=$ 18-27 years) were recruited from undergraduate classes at University of California-Los Angeles (UCLA). The ethnic composition of the sample reflected the diversity of students at UCLA: 47\% Asian, 16\% White, 16\% Filipino, 11\% Latino/Chicano, $5 \%$ Black, $5 \%$ other. All participants provided written consent in accordance with UCLA Institutional Review Board.

\section{Procedure}

Participants arrived at the lab along with a male confederate (the same one for each participant) who posed as another participant. The experimenter began by explaining that the purpose of the study was to understand how people make first impressions and their feelings about these impressions. Participants were told that, in the first part of the study, they would each complete an interview that would be recorded and later listened to by the other participant. In the second part of the study, they would each complete an fMRI scan as the other participant, who was outside the scanner, listened to and gave feedback on the interview. The person inside the scanner would view this feedback and rate his or her feelings in response.

After explaining the procedure, the participant and confederate were escorted into separate testing rooms and given questionnaire packets. The experimenter then interviewed the participant. Interview questions dealt with participant's opinions and personal characteristics such as "What is your best quality?," and "What are you most afraid of?" Each participant responded into a microphone so that the interview could be recorded; however, no recordings were made. After $10 \mathrm{~min}$ of questions, the interview was completed, and the participant was reminded that, during the scanning session, the other individual (confederate) would listen to the interview and rate his impressions of the participant. The experimenter then asked the participant to continue with the questionnaire packet while the other individual was ostensibly interviewed.

Approximately $10 \mathrm{~min}$ later, the experimenter returned and escorted the participant and confederate to the fMRI scanner. Before leaving the laboratory, the experimenter 
had the participant and confederate draw slips of paper to determine who would be scanned first. This drawing was rigged so that the real participant's name was always picked and he or she was scanned first. The confederate was never scanned.

Once at the fMRI scanning facility, the confederate waited in the lobby as the participant was set up in the scanner. After the participant was positioned in the scanner, the experimenter brought the confederate into the control room and reminded the participant and confederate of the task. Participants could hear that the confederate was in the room when he asked questions about the protocol. The computer interface that would be used to deliver the feedback was displayed to the real participant through a pair of goggles connected to a laptop computer (see Figure 1). The participant listened as the confederate was instructed to click on a descriptive feedback button every $10 \mathrm{sec}$ to give their honest impressions of how the participant was coming across in their interview. Participants were reminded to rate how they felt in response to seeing each feedback word using a four-button button box. On the basis of the previous behavioral paradigm (Leary et al., 1998, Experiment 4), participants reported their state selfesteem by rating "how they felt right now" on a 4-point scale from 1 (really bad) to 4 (really good). Participants made this rating after seeing each new feedback word in the fMRI scanner.

Once the participant was ready, he or she was scanned while viewing a video that was believed to be a live interface of the confederate's impressions of the participant's interview. The video showed a cursor moving over the 24 feedback buttons and clicking on a new word every
10 sec. Feedback adjectives were divided into one-third positive (e.g., "intelligent," "interesting"), one-third neutral ("practical," "talkative"), and one-third negative words (e.g., "boring," "insecure") based on pilot testing conducted with UCLA undergraduates $(n=74)$. These individuals were asked to rate how rejected or accepted they would feel if someone described them using each one of a list of adjectives. The final set of 24 adjectives (eight positive, eight neutral, and eight negative) was chosen based on these responses. Over the course of the video, participants viewed 15 presentations of each kind of adjective (thus certain words were repeated) in a pseudorandom order with the constraint that no more than two adjectives of the same valence could be presented consecutively (i.e., no more than two negative adjectives in a row). The feedback task was preceded and followed by a crosshair fixation $(10 \mathrm{sec})$. No participants suspected that this was a recording rather than a live interface.

In addition, before entering the scanner and immediately after exiting, participants rated how they felt about themselves on the 4-point scale used during the task itself. Prescan ratings were subtracted from postscan ratings to obtain a measure of changes in session-level self-esteem as a function of the feedback task.

\section{fMRI Data Acquisition}

Images were collected using a Siemens Trio 3-Tesla MRI scanner. First, a high-resolution structural scan (echoplanar T2-weighted spin-echo, TR $=4000 \mathrm{msec}$, TE $=$ $54 \mathrm{msec}$, matrix size $=128 \times 128, \mathrm{FOV}=20 \mathrm{~cm}, 36$ slices, 1.56-mm in-plane resolution, 3-mm thick) coplanar with
Figure 1. Picture of a sample feedback trial during the feedback task.

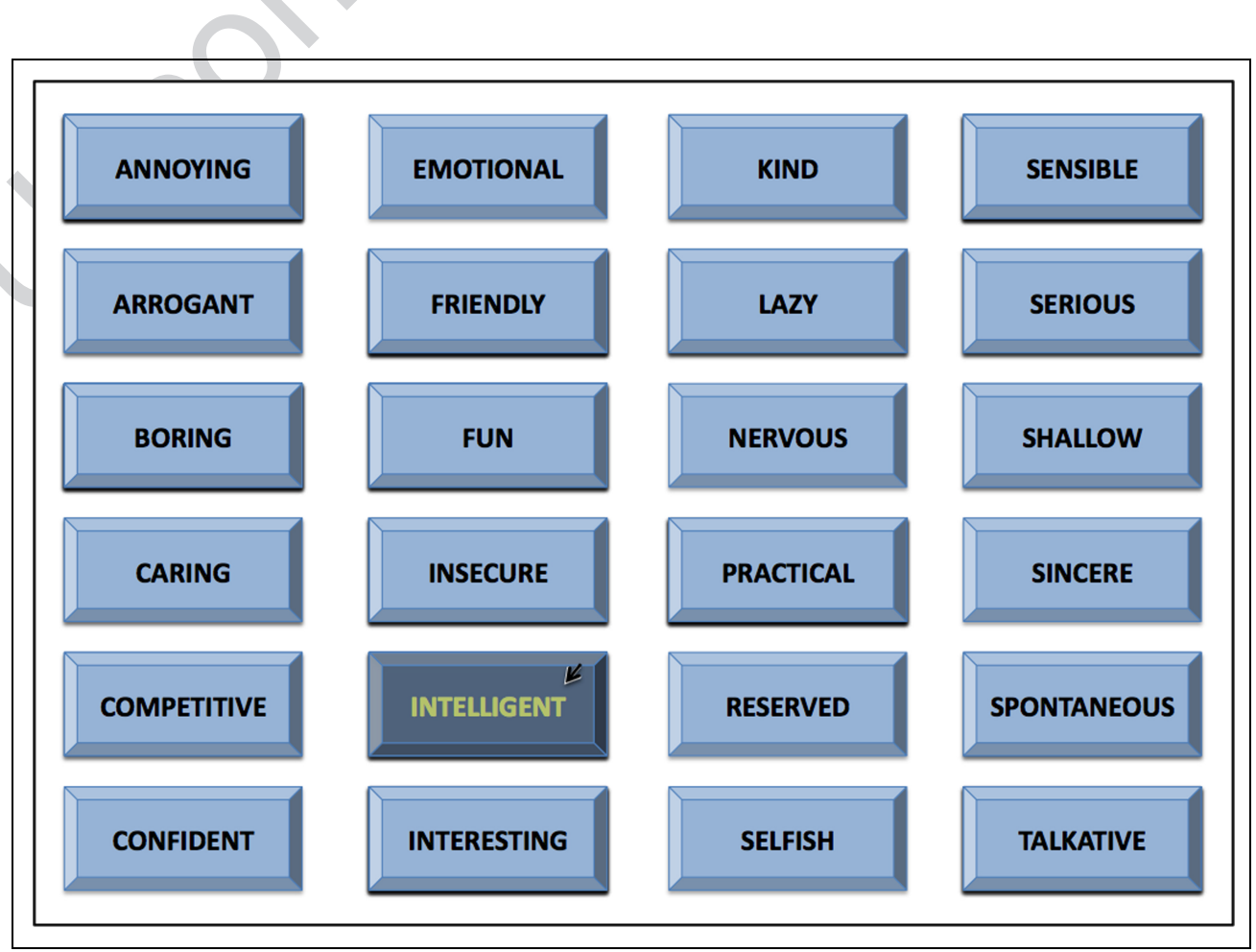


the functional scans was obtained for functional image registration during data preprocessing. Then, the feedback task was completed during a functional scan lasting $498 \mathrm{sec}$ (echo-planar T2*-weighted gradient-echo, TR = $3000 \mathrm{msec}, \mathrm{TE}=25 \mathrm{msec}$, flip angle $=90^{\circ}$, matrix size $=$ $64 \times 64,36$ axial slices, FOV $=20 \mathrm{~cm}, 3-\mathrm{mm}$ thick, 3-mm cubic voxel size, skip $=1 \mathrm{~mm}$ ).

\section{fMRI Data Analysis}

Neuroimaging data was preprocessed and analyzed using SPM5 (Wellcome Department of Cognitive Neurology, Institute of Neurology, London, United Kingdom). Preprocessing included image realignment to correct for head motion, normalization into a standard stereotactic space defined by the Montreal Neurological Institute (MNI) and the International Consortium for Brain Mapping, and spatial smoothing using an 8-mm Gaussian kernel, FWHM, to increase signal-to-noise ratio.

\section{Modeling of Contrasts}

The feedback task was modeled as a block design. The presentation of each feedback word and the subsequent $10 \mathrm{sec}$ were modeled as a block. To examine how self-reports of state self-esteem correlated with neural responses to each feedback word, we used a parametric modulator consisting of each participant's state self-esteem rating in response to each feedback word. These individual contrast images were then used in group-level, whole-brain analyses.

\section{Group-level, Whole-brain Analyses}

To examine how fluctuations in state self-esteem correlated with neural activity, we conducted whole-brain parametric modulation analyses. Contrast weights of -1 or 1 were entered to examine the neural regions that were negatively or positively, respectively, correlated with fluctuations in state self-esteem across the task. We also explored how neural activity during the feedback task related to changes in participants' session-level self-esteem from prescan to postscan. To do this, participants were divided in half based on whether their session-level selfesteem stayed the same or decreased from prescan to postscan. The correlations between neural activity and state self-esteem (parametric modulation) were then compared between these two groups. All whole-brain analyses were thresholded at $p<.001,10$ voxels (Lieberman \& Cunningham, 2009). All coordinates are reported in MNI format.

\section{RESULTS}

\section{Behavioral Results}

Consistent with previous research (Leary et al., 1998), participants reported significantly lower-state self-esteem following negative feedback trials $(M=1.81, S D=0.60)$ compared with either neutral $(M=3.13, S D=0.34$; $t(18)=10.55, p<.001, d=2.64)$ or positive feedback trials $(M=3.54, S D=0.37 ; t(18)=10.40, p<.001, d=$ $2.43)$. State self-esteem was also significantly lower following neutral compared with positive feedback trials $(t(18)=$ 5.51, $p<.001, d=1.25$ ).

On average, participants reported feeling significantly lower-session-level self-esteem after the scanning session ( $M=3.0, S D=0.67$ ) compared with before the scanning session $(M=3.37, S D=0.60 ; t(18)=2.67, p<.05, d=$ $0.62)$. Specifically, 8 participants showed a drop in sessionlevel self-esteem, 10 participants showed no change, and 1 participant showed an increase. This asymmetry may have occurred because of the fact that two thirds of the feedback words were negative or neutral, and previous research suggests that people tend to interpret neutral feedback as rejecting (Leary et al., 1998). Thus, although we balanced the number of negative, neutral, and positive adjectives, participants may have subjectively experienced the words as more rejecting than accepting, leading many more participants to show an overall drop in session-level self-esteem rather than an increase.

\section{Neuroimaging Results}

Because our primary focus was on participants' individualized response to each feedback word, as opposed to responses to feedback words that were determined a priori (through pilot testing) to be positive, neutral, or negative, we examined how moment-to-moment fluctuations in state self-esteem correlated with neural responses to each feedback word across the task. To examine this, we conducted parametric modulation analyses exploring which neural regions had moment-to-moment changes in neural activity that correlated negatively or positively with moment-to-moment changes in state self-esteem.

\section{Negative Correlations with State Self-esteem}

Consistent with previous research on social rejection to the extent that participants reported lower-state self-esteem on a trial-by-trial basis, they showed greater activity in regions involved in processing social rejection, including the bilateral anterior insula and the dACC (Figure 2 and Table 1). Moreover, participants who reported lower-state self-esteem also showed greater activity in regions involved in mentalizing or thinking about the thoughts of others, including the DMPFC (BA 9/BA 10; Figure 2) and pSTS (Table 1). No other regions correlated negatively with state self-esteem.

\section{Positive Correlations with State Self-esteem}

State self-esteem correlated positively with activity in several neural regions (see Table 2). Higher levels of state self-esteem were associated with greater activity in bilateral 


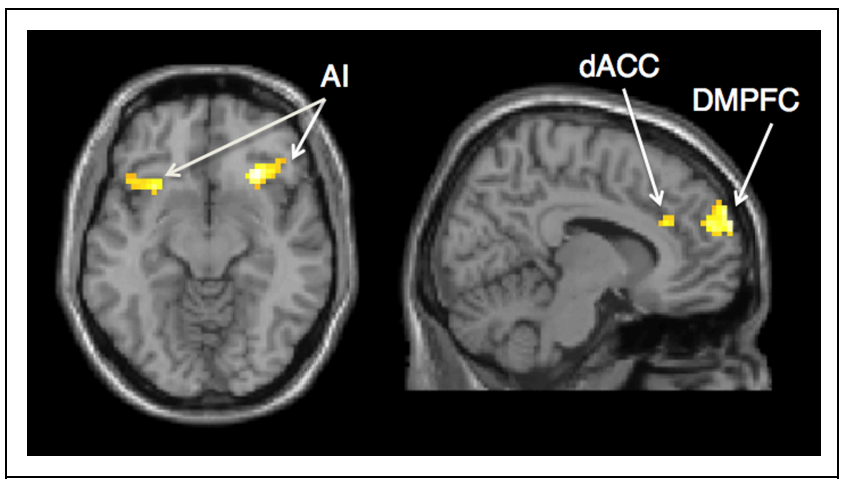

Figure 2. Neural regions that correlated negatively with self-reported state self-esteem (based on parametric modulation).

posterior insula, a region that has been shown to respond to positive stimuli, such as pleasant touch (Björnsdotter, Löken, Olausson, Vallbo, \& Wessberg, 2009; Olausson et al., 2002) and monetary rewards (Fujiwara, Tobler, Taira, Iijima, \& Tsutsui, 2009). State self-esteem also correlated positively with several regions within the precuneus-a region linked with mentalizing, self-referential thought, and reward (Lieberman, 2010; Fujiwara et al., 2009; Cavana \& Trimble, 2006; Northoff \& Bermpohl, 2004). However, state self-esteem did not correlate positively with activity in the VS or subACC.

\section{Between-group Differences Due to Changes in Session-level Self-esteem}

Next, we wanted to investigate the kind of neural activity that differentiated subjects whose session-level self-esteem decreased following the feedback task versus those whose session-level self-esteem did not. Participants were divided into two groups: those whose session-level self-esteem decreased $(n=8)$ versus those whose session-level selfesteem stayed the same or improved $(n=11)$. To examine differences in the relationship between neural activity

Table 1. Regions that Correlated Negatively with State Self-esteem (Based on Parametric Modulation)

\begin{tabular}{|c|c|c|c|c|c|c|c|c|}
\hline $\begin{array}{l}\text { Anatomical } \\
\text { Region }\end{array}$ & $B A$ & & $x$ & $y$ & $z$ & $t$ & $k$ & $p$ \\
\hline \multirow{2}{*}{$\begin{array}{r}\text { Anterior } \\
\text { insula }\end{array}$} & & $\mathrm{R}$ & 30 & 27 & -9 & 6.65 & 108 & $<.0001$ \\
\hline & & $\mathrm{L}$ & -30 & 18 & -9 & 5.46 & 46 & $<.0001$ \\
\hline dACC & 32 & $\mathrm{R}$ & 9 & 30 & 24 & 3.63 & 12 & $<.0001$ \\
\hline DMPFC & $9 / 10$ & $\mathrm{R}$ & 9 & 66 & 24 & 6.05 & 230 & $<.0001$ \\
\hline pSTS & 22 & $\mathrm{R}$ & 48 & -27 & -3 & 4.85 & 49 & $<.0001$ \\
\hline
\end{tabular}

BA refers to putative Brodmann's Area; L and R refer to left and right hemispheres; $x, y$, and $z$ refer to MNI coordinates in the left-right, anteriorposterior, and interior-superior dimensions, respectively; $t$ refers to the $t$ score at those coordinates (local maxima); $k$ refers to the number of voxels in each significant cluster.
Table 2. Regions that Correlated Positively with State Self-esteem (Based on Parametric Modulation)

\begin{tabular}{|c|c|c|c|c|c|c|c|c|}
\hline $\begin{array}{l}\text { Anatomical } \\
\text { Region }\end{array}$ & $B A$ & & $x$ & $y$ & $z$ & $t$ & $k$ & $p$ \\
\hline \multirow[t]{3}{*}{ Posterior insula } & & $\mathrm{R}$ & 33 & -15 & 27 & 7.97 & 51 & $<.0001$ \\
\hline & & $\mathrm{L}$ & -36 & -21 & 21 & 6.10 & 257 & $<.0001$ \\
\hline & & $\mathrm{R}$ & 45 & -15 & 12 & 4.66 & 10 & $<.0001$ \\
\hline Midinsula & & $\mathrm{R}$ & 33 & 9 & 12 & 4.22 & 12 & $<.0001$ \\
\hline Anterior insula & & $\mathrm{L}$ & -21 & 27 & 15 & 4.89 & 20 & $<.0001$ \\
\hline \multirow[t]{2}{*}{ Precuneus } & 7 & $\mathrm{R}$ & 15 & -42 & 75 & 5.21 & 50 & $<.0001$ \\
\hline & 7 & $\mathrm{~L}$ & -15 & -42 & 60 & 4.66 & 78 & $<.0001$ \\
\hline \multirow[t]{5}{*}{ SMA } & 6 & $\mathrm{R}$ & 15 & -3 & 48 & 5.81 & 45 & $<.0001$ \\
\hline & 6 & $\mathrm{~L}$ & -18 & -6 & 60 & 5.38 & 74 & $<.0001$ \\
\hline & 6 & $\mathrm{R}$ & 15 & -21 & 63 & 5.14 & 36 & $<.0001$ \\
\hline & 6 & $\mathrm{R}$ & 18 & -33 & 57 & 4.90 & 12 & $<.0001$ \\
\hline & 6 & $\mathrm{~L}$ & -21 & -18 & 51 & 4.68 & 10 & $<.0001$ \\
\hline \multirow[t]{2}{*}{ Premotor cortex } & 6 & $\mathrm{R}$ & 60 & 3 & 12 & 4.62 & 29 & $<.0001$ \\
\hline & 6 & $\mathrm{R}$ & 24 & 6 & 30 & 4.75 & 10 & $<.0001$ \\
\hline Occipital cortex & 18 & $\mathrm{R}$ & 18 & -75 & -9 & 5.42 & 478 & $<.0001$ \\
\hline $\begin{array}{l}\text { Somatosens. } \\
\text { cortex }\end{array}$ & $1 / 2 / 3$ & $\mathrm{R}$ & 24 & -21 & 36 & 5.12 & 12 & $<.0001$ \\
\hline Inf. parietal lobule & 40 & $\mathrm{R}$ & 57 & -24 & 18 & 4.51 & 22 & $<.0001$ \\
\hline
\end{tabular}

BA refers to putative Brodmann's Area; L and R refer to left and right hemispheres; $x, y$, and $z$ refer to MNI coordinates in the left-right, anteriorposterior, and interior-superior dimensions, respectively; $t$ refers to the $t$ score at those coordinates (local maxima); $k$ refers to the number of voxels in each significant cluster. The following abbreviations are used for the names of specific regions: somatosens. cortex $=$ somatosensory cortex; inf. parietal lobule $=$ inferior parietal lobule.

and moment-to-moment fluctuations in state self-esteem between these two groups, we conducted between-group parametric modulation analyses. Only one neural region differentially correlated with state self-esteem as a function of changes in session-level self-esteem-the medial pFC (MPFC; BA 10; $-12,54,18, t=5.02, k=16, p<.0001)^{1}-$ a region involved in self-referential thought (Lieberman, 2010; Northoff \& Bermpohl, 2004; Kelley et al., 2002). To further clarify these results, we extracted parameter estimates from this cluster separately for positive, neutral, and negative feedback trials (based on categories established during pilot testing). These analyses demonstrated that individuals whose session-level self-esteem decreased after the feedback task (versus those whose self-esteem did not) showed significantly more MPFC activation in response to negative feedback words $(t(17=1.72, p=.05, d=0.77$; Figure 3$)$. Thus, these individuals may have been processing these negative words as more self-relevant or encoding this feedback more deeply, and this deeper encoding may have led to the reductions in session-level self-esteem. No differences in MPFC activation were found between the 
Figure 3. Bar graph depicting neural activity in the MPFC in response to positive, neutral, and negative feedback trials as a function of whether session-level state self-esteem decreased from pretask to posttask versus stayed the same (or increased).

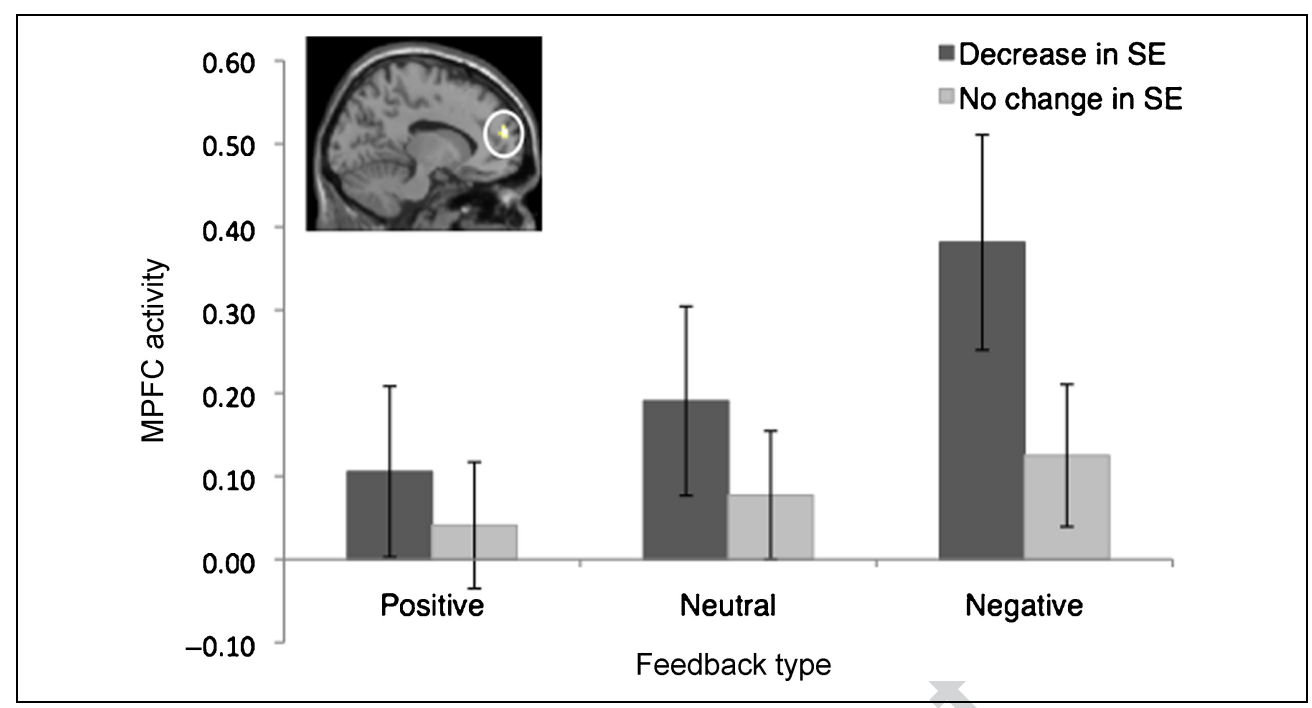

two groups in response to neutral or positive feedback words $(p s>.20)$.

\section{DISCUSSION}

Although a century of research has demonstrated that people strive to maintain self-esteem, considerably less attention has focused on why. The current study aimed to investigate one account of why individuals strive for self-esteemsociometer theory. According to this theory, state selfesteem is an internal, psychological response to people's perceptions of the degree to which they are valued and accepted versus devalued and rejected by others (Leary, 2006a, 2006b; Leary \& Baumeister, 2000). If self-feelings are, in fact, a "proxy" for perceived acceptance or rejection (Leary et al., 1995), then brain regions associated with processing acceptance and rejection should play an important role in state self-esteem. The current results support this idea. Reductions in state self-esteem were associated with greater activity in the bilateral anterior insula and the dACC, the same regions that have been shown to be activated by experiences of interpersonal rejection and to correlate with self-reported distress in response to these experiences (DeWall et al., 2010, in press; Masten et al., 2009; Onoda et al., 2009; Eisenberger, Taylor, et al., 2007; Kross et al., 2007; Eisenberger et al., 2003). Thus, in line with sociometer theory, neural regions that have been shown to process the distress of social rejection were also directly associated with reduced feelings of state self-esteem in response to negative feedback.

Lower-state self-esteem was also associated with greater activity in regions involved in mentalizing or understanding the minds of others, such as the DMPFC (BA 9/BA 10) and pSTS. Given that participants were viewing what they believed was another person's impressions of them, activity in these areas is understandable (Lieberman, 2010; Mitchell, 2009; Frith \& Frith, 2006). However, it is not yet clear why greater activity in the DMPFC and pSTS corre- lated with reductions, rather than increases, in state selfesteem. One possibility is that negative social feedback engenders not only reductions in state self-esteem but also a greater attempt at figuring out the reasons for the negative feedback, which might involve a greater focus on processing the minds or intentions of others. Indeed, negative feedback has been shown to have a greater impact and to be processed more thoroughly than positive feedback (Baumeister, Bratslavsky, Finkenauer, \& Vohs, 2001; Abele, 1985; Weiner, 1985). Thus, regions like the DMPFC and pSTS, often involved in mentalizing, may be associated with negative-state self-esteem because of their role in processing the psychological meaning of the underlying negative social feedback. Future work, however, will be needed to further understand the role of the DMPFC and pSTS in reduced state self-esteem.

With regard to positive correlations with state selfesteem, higher-state self-esteem did not correlate positively with neural regions previously associated with social acceptance or positive social feedback, such as the VS (Izuma et al., 2008) or the subACC (Somerville et al., 2006). There are several possible reasons for this. First, research showing VS activity in response to positive social feedback included only positive and neutral feedback trials, but no negative feedback trials (Izuma et al., 2008). The fact that approximately one third of the feedback trials in the current study were negative may have reduced the rewarding experience associated with positive social feedback, thus reducing any VS responses. Second, in both previous studies on social acceptance (Somerville et al., 2006) and positive social feedback (Izuma et al., 2008), the feedback came from a series of different individuals, whereas in the current study, all of the feedback came from the same individual. Receiving positive feedback from some individuals and negative feedback from others, as has been done previously, may allow subjects to engage strategies that discount the opinions of those who provide negative feedback and augment the opinions of those who 
provide positive feedback. However, receiving both positive and negative feedback from the same individual, as was done here, may be a more unnerving and potentially less positive experience because of the greater difficulty involved in logically discounting the negative and augmenting the positive feedback from the same individual.

We did, however, find that higher-state self-esteem correlated with greater activity in several regions of the posterior insula. This is interesting because, in addition to this region being involved in responding to rewarding stimuli (Fujiwara et al., 2009), it has been shown to play a role in processing pleasant physical touch (e.g., soft stroking of the skin) as opposed to other kinds of touch (Björnsdotter et al., 2009; Olausson et al., 2002). Although speculative, it is possible that receiving positive social feedback was interpreted as "emotionally touching" and that these kinds of positive experiences may be processed by some of the same regions that process pleasant physical touch.

Analysis of changes in session-level self-esteem from before to after the fMRI scan revealed that participants whose session-level self-esteem decreased after the feedback task showed more MPFC activation (BA 10) in response to negative feedback words than those whose self-esteem increased or stayed the same. Given that MPFC activation is associated with reflecting upon one's personal traits and encoding self-relevant information (Lieberman, 2010; Amodio \& Frith, 2006; Frith \& Frith, 2006; Macrae, Moran, Heatherton, Banfield, \& Kelley, 2004; Kelley et al., 2002), this finding may suggest that participants whose sessionlevel self-esteem declined processed these negative feedback words as more self-relevant or encoded the negative feedback more deeply. Thus, greater self-relevant processing or deeper encoding of these negative feedback words may have led these individuals to feel worse about themselves after the scan. Because the MPFC has not previously been shown to be sensitive to valence (Moran, Macrae, Heatherton, Wyland, \& Kelley, 2006), further research will be needed to determine whether this region plays a more general role in changes in session-level self-esteem based on the type of feedback (positive, negative) that is more deeply or more self-referentially encoded. For example, had we observed a group of participants who showed an increase in session-level self-esteem, these individuals may have displayed greater MPFC to positive feedback. Thus, the MPFC activity may be less directly associated with decreases in session-level self-esteem and more strongly related to changes in session-level self-esteem as a function of the type of information that is being more deeply processed.

The fact that different neural regions related to state selfesteem versus changes in session-level self-esteem fits with previous research showing that moment-to-moment reports of affective experience and retrospective reports of affective experience do not necessarily correspond (Eisenberger, Gable, \& Lieberman, 2007; Redelmeier \& Kahneman, 1996; Frederickson \& Kahneman, 1993; Kahneman, Frederickson, Schreiber, \& Redelmeier, 1993). In the present study, moment-to-moment reports of state self-esteem mapped on more closely to regions involved in distressing emotional experience (dACC, anterior insula), whereas changes in session-level self-esteem (which could be conceptualized as a more retrospective assessment) relied on regions involved in self-referential and autobiographical memory encoding (MPFC; Eisenberger, Gable, et al., 2007; Cabeza et al., 2004; Macrae et al., 2004). Given this difference, it would be interesting to further investigate individual differences that are more closely related to each neural subsystem.

Finally, it should be noted that there were several limitations of this study. First, participants viewed each feedback word for $10 \mathrm{sec}$, which is a fairly long period. Because of this, it is possible that the effects of the feedback may have been diluted because of the participants thinking about other things. However, the fact that we still observed neural activity that correlated with changes in state self-esteem suggests that the neural activity during this response window was still meaningful. In addition, it should be noted that all participants received feedback from a single male confederate. Thus, although all participants received feedback from the same individual, we cannot examine the effect of confederate gender on the outcomes reported here.

In summary, the findings reported here begin to identify the neural regions that play a role in moment-to-moment fluctuations in self-related feelings. Consistent with sociometer theory, we found that the same regions that have been shown to be involved in responding to social rejection are also involved in producing low-state self-esteem. Moreover, we found some differences in the neural regions associated with moment-to-moment changes in state self-esteem (dACC, anterior insula) and those that relate to more long-lasting changes in state self-esteem (MPFC). Together, these data may have important implications for understanding the nature of our feelings about ourselves and how these feelings are intrinsically tied to beliefs about how we are perceived or evaluated by others.

\section{Acknowledgments}

We thank the UCLA Brainmapping Center, Brian Vickers, and Tamara Sussman for their assistance with this project. We also thank Matthew Lieberman for useful feedback on drafts of this manuscript.

Reprint requests should be sent to Naomi I. Eisenberger, Department of Psychology, 1285 Franz Hall, University of California, Los Angeles, CA 90095-1563, or via e-mail: neisenbe@ucla.edu.

\section{Note}

1. This MPFC activation (BA 10) was more inferior than the DMPFC activation (BA 9/BA 10) that correlated negatively with state self-esteem and only overlapped with this DMPFC activation by one voxel.

\section{REFERENCES}

Abele, A. (1985). Thinking about thinking: Casual, evaluative, and finalistic cognitions about social situations. European Journal of Social Psychology, 15, 315-332. 
Amodio, D. M., \& Frith, C. D. (2006). Meeting of minds: The medial frontal cortex and social cognition. Nature Reviews Neuroscience, 7, 268-277.

Baumeister, R. F., Bratslavsky, E., Finkenauer, C., \& Vohs, K. D. (2001). Bad is stronger than good. Review of General Psychology, 5, 323-370.

Björnsdotter, D., Löken, L., Olausson, H., Vallbo, A., \& Wessberg, J. (2009). Somatotopic organization of gentle touch processing in the posterior insular cortex. Journal of Neuroscience, 29, 9314-9320.

Burklund, L., Eisenberger, N. I., \& Lieberman, M. D. (2007). The face of rejection: Rejection sensitivity moderates dorsal anterior cingulated cortex activity to disapproving facial expressions. Social Neuroscience, 2, 238-253.

Cabeza, R., Prince, S. E., Daselaar, S. M., Greenberg, D. L., Budde, M., Dolcos, F., et al. (2004). Brain activity during episodic retrieval of autobiographical and laboratory events: An fMRI study using a novel photo paradigm. Journal of Cognitive Neuroscience, 16, 1583-1594.

Cavana, A. E., \& Trimble, M. R. (2006). The precuneus: A review of its functional anatomy and behavioural correlates. Brain, 129, 564-583.

DeWall, C. N., MacDonald, G., Webster, G. D., Masten, C. L., Baumeister, R. F., Powell, C., et al. (2010). Tylenol reduces social pain: Behavioral and neural evidence. Psychological Science, 21, 931-937.

DeWall, C. N., Masten, C. L., Powell, C., Combs, D., Schurtz, D. R., $\&$ Eisenberger, N. I. (in press). Does the pain of rejection depend on attachment style? An fMRI study. Social Cognitive and Affective Neuroscience.

Eisenberger, N. I., Gable, S. L., \& Lieberman, M. D. (2007). fMRI responses relate to differences in real-world social experiences. Emotion, 7, 745-754.

Eisenberger, N. I., Lieberman, M. D., \& Williams, K. D. (2003). Does rejection hurt? An fMRI study of social exclusion. Science, 302, 290-292.

Eisenberger, N. I., Taylor, S. E., Gable, S. L., Hilmert, C. J., \& Lieberman, M. D. (2007). Neural pathways link social support to attenuated neuroendocrine stress responses. Neuroimage, 35, 1601-1612.

Frederickson, B. L., \& Kahneman, D. (1993). Duration neglect in retrospective evaluations of affective episodes. Journal of Personality and Social Psychology, 65, 45-55.

Frith, C. D., \& Frith, U. (2006). The neural basis of mentalizing. Neuron, 50, 531-534.

Fujiwara, J., Tobler, P. N., Taira, M., Iijima, T., \& Tsutsui, K.-I. (2009). A parametric relief signal in human ventrolateral prefrontal cortex. Neuroimage, 44, 1163-1170.

Izuma, K., Saito, D. N., \& Sadato, N. (2008). Processing of social and monetary rewards in the human striatum. Neuron, 58, 284-294.

James, W. (1890). The principles of psychology. New York: Henry Holt and Company.

Kahneman, D., Frederickson, B. L., Schreiber, C. A., \& Redelmeier, D. A. (1993). When more pain in preferred to less: Adding a better end. Psychological Science, 4, 401-405.

Kelley, W. M., Macrae, C. N., Wyland, C. L., Caglar, S., Inati, S., \& Heatherton, T. F. (2002). Finding the self? An event-related fMRI study. Journal of Cognitive Neuroscience, 14, 785-794.

Knutson, B., \& Cooper, J. C. (2005). Functional magnetic resonance imaging of reward prediction. Current Opinion in Neurology, 18, 411-418.

Kross, E., Egner, T., Ochsner, K., Hirsch, J., \& Downey, G. (2007). Neural dynamics of rejection sensitivity. Journal of Cognitive Neuroscience, 19, 945-956.

Leary, M. (1999). Making sense of self-esteem. Current Directions in Psychological Science, 8, 32-35.
Leary, M. (2006a). Motivational and emotional aspects of the self. Annual Review of Psychology, 58, 317-344.

Leary, M. R. (2006b). Sociometer theory and the pursuit of relational value: Getting to the root of self-esteem. European Review of Social Psychology, 16, 75-111.

Leary, M. R., \& Baumeister, R. F. (2000). The nature and function of self-esteem: Sociometer theory. Advances in Experimental Social Psychology, 32, 1-62.

Leary, M. R., \& Downs, D. L. (1995). Interpersonal functions of the self-esteem motive: The self-esteem system as a sociometer. In M. H. Kernis (Ed.), Efficacy, agency, and self-esteem (pp. 123-144). New York: Plenum Press.

Leary, M. R., Haupt, A. L., Strausser, K. S., \& Chokel, J. T. (1998). Calibrating the sociometer: The relationship between interpersonal appraisals and state self-esteem. Journal of Personality and Social Psychology, 74, 1290-1299.

Leary, M. R., Tambor, E. S., Terdal, S. K, \& Downs, D. L. (1995). Self-esteem as an interpersonal monitor: The sociometer hypothesis. Journal of Personality and Social Psychology, $68,518-530$.

Lieberman, M. D. (2010). Social cognitive neuroscience. In S. T. Fiske, D. T. Gilbert, \& G. Lindzey (Eds.), Handbook of social psychology (5th ed., pp. 143-193). New York: McGraw-Hill.

Lieberman, M., \& Cunningham, W. A. (2009). Type I and type II error concerns in fMRI research: Re-balancing the scale. Social Cognitive Neuroscience, 4, 423-428.

Macrae, C. N., Moran, J. M., Heatherton, T. F., Banfield, J. F., \& Kelley, W. M. (2004). Medial prefrontal activity predicts memory for self. Cerebral Cortex, 14, 647-654.

Masten, C. L., Eisenberger, N. I., Borofsky, L., Pfeifer, J. H., McNealy, K., Mazziotta, J. C., et al. (2009). Neural correlates of social exclusion during adolescence: Understanding the distress of peer rejection. Social Cognitive and Affective Neuroscience, 4, 143-157.

Mitchell, J. P. (2009). Inferences about mental states. Philosophical Transactions of the Royal Society, Biological Sciences, 364, 1309-1316.

Moran, J. M., Macrae, C. N., Heatherton, T. F., Wyland, C. L., \& Kelley, W. M., (2006). Neuroanatomical evidence for distinct cognitive and affective components of self. Journal of Cognitive Neuroscience, 18, 1586-1594.

Northoff, G., \& Bermpohl, F. (2004). Cortical midline structures and the self. Trends in Cognitive Sciences, 8, 102-107.

Olausson, H., Lamarre, Y., Backlund, H., Morin, C., Wallin, B. G., Starck, G., et al. (2002). Unmeyelinated tactile afferents signal touch and project to insular cortex. Nature, 5, 900-904.

Onoda, K., Okamoto, Y., Nakashima, K., Nittono, H., Ura, M., \& Yamawaki, S. (2009). Decreased ventral anterior cingulate cortex activity is associated with reduced social pain during emotional support. Social Neuroscience, 4, 443-454.

Onoda, K., Okamoto, Y., Nakashima, K., Nittono, H., Yoshimura, S., Yamawaki, S., et al. (2010). Does low self-esteem enhance social pain? The relationship between trait self-esteem and anterior cingulate cortex activation induced by ostracism. Social Cognitive and Affective Neuroscience, 5, 385-391.

Redelmeier, D. A., \& Kahneman, D. (1996). Patients' memories of painful medical treatments: Real-time and retrospective evaluations of two minimally evasive procedures. Pain, 66, 3-8.

Somerville, L. H., Heatherton, T. F., \& Kelley, W. M. (2006). Anterior cingulate cortex responds differentially to expectancy violation and social rejection. Nature Neuroscience, 9, 1007-1008.

Somerville, L. H., Kelley, W. M., \& Heatherton, T. F. (2010). Selfesteem modulates medial prefrontal cortical responses to evaluative social feedback. Cerebral Cortex, 20, 3005-3013.

Weiner, B. (1985). "Spontaneous" causal thinking. Psychological Bulletin, 97, 74-84. 


\section{AUTHOR QUERIES}

\section{AUTHOR PLEASE ANSWER ALL QUERIES}

During the preparation of your manuscript, the questions listed below arose. Kindly supply the necessary information.

1. Please update DeWall et al., in press if it has already been published.

2. Define TR, TE, FOV.

3. Please check authors of Masten et al., 2009.

\section{END OF ALL QUERIES}

